Intensivmed 2010 • 47:550-551

DOI 10.1007/s00390-010-0229-7

Online publiziert: 30 . Oktober 2010

๑) Springer-Verlag 2010

\author{
S. Rosseau ${ }^{1} \cdot$ M. Joannidis ${ }^{2} \cdot$ U. Janssens ${ }^{3}$ \\ ${ }^{1}$ Medizinische Klinik m.S. Infektiologie und Pneumologie, Charité \\ Zentrum für ambulante Beatmungsmedizin, Campus Mitte, Berlin \\ ${ }^{2}$ Universitätsklinik für Innere Medizin I, Innsbruck \\ ${ }^{3}$ Innere Medizin, St.-Antonius-Hospital, Eschweiler
}

\title{
CME - nun auch in der Intensivmedizin und Notfallmedizin
}

für Neurologische Intensiv- und Notfallmedizin, der Arbeitsgemeinschaft Intensivmedizin im Berufsverband Deutscher Internisten sowie der Deutschen Interdisziplinären Vereinigung für Intensivmedizin und Notfallmedizin stetig weiterentwickelt. Mittlerweile werden Themenhefte gestaltet und herausgegeben, die sich schwerpunktmäßig einem besonderen Inhalt der Intensivmedizin widmen. Dieses neue Konzept erfreut sich einer hohen Akzeptanz innerhalb der Leserschaft. medical education") sowie kontinuierliche berufliche Kompetenzerhaltung und -entwicklung $(\mathrm{CPD}=$, ,ontinuing professional development") erforderlich. Ärztliche Kompetenz ist jedoch mehr als der Erwerb von Informationen. Es geht im Kern um Wissen und Umsetzung dieses Wissens in ärztliche Fähigkeiten, Fertigkeiten und Haltungen. Ein wichtiger Baustein für den Kompetenzerhalt ist der ,freiwillige Fortbildungsnachweis der Ärztekammern."

Die Zeitschrift Intensivmedizin und Notfallmedizin hat sich als Organ der Deutschen Gesellschaft für Internistische Intensivmedizin und Notfallmedizin, der Österreichischen Gesellschaft für Internistische und Allgemeine Intensivmedizin, der Deutschen Gesellschaft für Innere Medizin, der Deutschen Gesellschaft

\section{- Eine horizontal wie vertikal breit angelegte Fortbildung ist für die Qualität unserer täglichen anspruchsvollen Arbeit in der Intensivmedizin unerlässlich.}

Wir greifen dabei auf lokale, nationale, aber auch internationale Kongresse der Fachgesellschaften zurück, die dort erworbenen Kenntnisse gehören jedoch vertieft und müssen immer wieder nachgearbeitet und aufgefrischt werden. Vor diesem Hintergrund versteht sich die neue CME-Rubrik der Zeitschrift. Zukünftig werden aktuelle Leitlinien und Empfehlungen, aber auch Therapieverfahren und diagnostische Methoden in dieser Rubrik strukturiert durch Experten auf diesem
Gebiet aufgearbeitet. Wir als Herausgeber sind sehr froh, dass der Verlag sich entschlossen hat, auch dieser Fortbildungsart einen Raum zu bieten, und glauben, dass damit die Attraktivität, aber auch die Qualität unserer Zeitschrift deutlich zunehmen wird.

Der interdisziplinäre Charakter der Zeitschrift wird sich auch durch die Auswahl der Themen und der Autoren widerspiegeln: Im ersten Beitrag der CME-Rubrik fasst M. Buerke die aktuellen Empfehlungen zur Diagnostik und Therapie des infarktbedingten kardiogenen Schocks zusammen. Die weltweit ersten Leitlinien zu dieser Thematik wurden jüngst unter der Federführung von Professor Werdan, Halle, veröffentlicht und können auf der Homepage der AWMF eingesehen werden (http://leitlinien.net/). Sie stellen die Grundlage dieser ersten CME-zertifizierten Weiterbildung dar. In folgenden Ausgaben werden Themen wie Sepsis, Analgosedierung, Fehler in der Intensivmedizin, Überleitmanagement, Hypothermie und Pneumonie aufgegriffen.

Die Herausgeber sind natürlich sehr an Ihrer Meinung interessiert: Kritik, Anregungen, aber sicher auch Lob sind jederzeit mehr als nur willkommen. Bitte neh- 
men Sie mit uns für diesen Fall Kontakt auf.

Das Herausgeberteam freut sich sehr auf diese Beiträge und wünscht Ihnen, den Leserinnen und Lesern, eine spannende Lektüre.

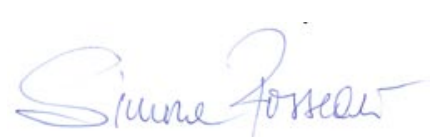

Dr. Simone Rosseau

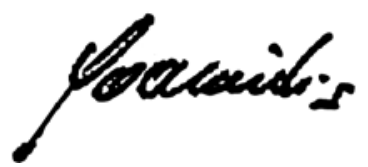

Prof. Dr. Michael Joannidis

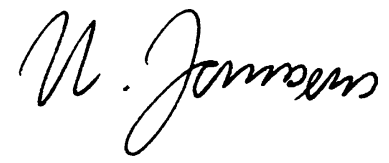

Prof. Dr. Uwe Janssens

\section{Korrespondenzadressen}

\section{Dr. S. Rosseau}

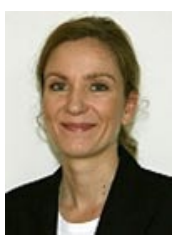

Medizinische Klinik m.S. Infektiologie und

Pneumologie,

Charité Zentrum für ambulante

Beatmungsmedizin,

Campus Mitte

Charitéplatz 1, 10117 Berlin

simone.rosseau@charite.de

\section{Prof. Dr. M. Joannidis}

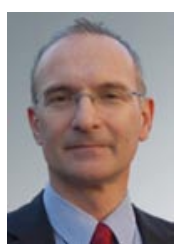

Universitätsklinik für Innere

Medizin I

Anichsstraße 35,

6020 Innsbruck

Österreich

Michael.Joannidis@i-med.ac.at

Prof. Dr. U. Janssens

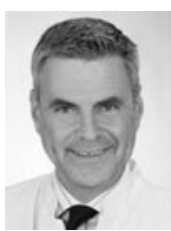

Innere Medizin,

St.-Antonius-Hospital

Dechant-Deckers-Straße

8, 52249 Eschweiler

uwe.janssens@sah-

eschweiler.de

\section{CME.springer.de}

\section{Springer Medizin}

\section{Abonnenten sammeln kostenlos CME-Punkte}

Ihre Fachzeitschrift bietet Ihnen in jeder Ausgabe einen Fortbildungsbeitrag, der mit 3 CME-Punkten zertifiziert ist. Wenn Sie Abonnent sind, ist die Teilnahme auf CME.springer.de für Sie kostenlos.

\section{So einfach geht's:}

$>$ 1. Registrieren

Um Fortbildungseinheiten auf

CME.springer.de bearbeiten zu können, müssen Sie sich einmalig mit Ihrer Abonummer registrieren. Sind Sie bereits registriert, können Sie unter Meine Daten $>$ Abo hinzufügen ihre Abonummer hinterlegen. Sie finden diese auf Ihrem Adressetikett.

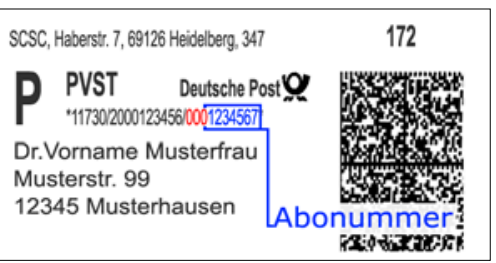

\section{> 2. Beitrag auswählen} Wählen Sie die gewünschte Fortbildungseinheit aus und Klicken Sie auf teilnehmen. Sie können nun den Zeitschriftenbeitrag als PDF herunterladen und lesen oder direkt den Fragebogen beantworten.

7. 3. CME-Punkte sammeln

Zu jedem Beitrag gehört ein Fragebogen mit 10 CME-Fragen. Mit 7 richtigen Antworten haben Sie bestanden und erhalten

Ob Sie Ihr Wissen auffrischen möchten, oder sich auf die Facharztprüfung vorbereiten - mit den CME-Beiträgen von Springer sind Sie immer auf dem neuesten Stand. umgehend eine Teilnahmebescheinigung! 